\title{
Bionic Technology Research Study Based on the Non-smooth Surface Morphology of the Mosquito Mouthparts
}

\author{
Jingchun Wang ${ }^{1}$, Yu Chen ${ }^{2 *}$, Luquan Ren ${ }^{1}$, Yinwu $\mathrm{Li}^{1}$ and Changhai Zhou ${ }^{1}$ \\ ${ }^{1}$ College of Biological and Agricultural Engineering, Jilin University, China \\ ${ }^{2}$ Engineering training center, Jilin University, China \\ ${ }^{\star}$ The corresponding author
}

Keywords: Non-smooth Function; Reducing Resistance; Engineering Bionics; Needles; Mouthpart

\begin{abstract}
Using the stereomicroscope and scanning electron microscope, the mosquito mouthpart was observed. The surface morphology of its six mouth needles shows that the upper jaw and lower jaw in mosquito mouthpart, which present typical non-smooth surface morphology, play a role in piercing skins and they are the main organ to do this. The end of the upper jaw is broad like the shape of knife, and there are 25-30 thin saw teeth inside. The non-smooth morphology model of mosquito mouthpart was put in the rear of the injector model, and the rate of reducing resistance can reach as much as $44.5 \%$ through Experience [1].
\end{abstract}

\section{Introduction}

In order to meet the needs of their own survival, creatures in the nature use different ways of pray. For example, mosquitoes and cicadas have piercing-sucking mouthparts which have special structure and function. The research shows that mosquito's "mouth" (beak) is highly serrated and only its front piercing surface is very smooth, unlike needles which usually have smooth surface. Therefore, there is only a small point of contact when the mouth (beak) of mosquito penetrates into the skin of human or animal and suck blood. The nerve, consequently, is not very sensitive to the penetration. People feel itching simply because that when the mosquito is sucking blood, it can secrete a kind of saliva which can make the people feel itching. In medicine, itching should also be classified as Slightly-pain and the sensibility of the nervous is not so high to feel the faint pain $[2,3]$.

The specific function of the mosquito's mouthpart is determined by its special structure. Studying the surface morphology of mosquito mouthpart is the foundation for the engineering bionic research in medical apparatus, which is significant to develop injection alleviating the pain.

\section{Material and Methods}

Mosquito belongs to arthropod, insect, dipteral, long-horned suborder, mosquitoes. The mouths of most kinds of the female mosquitoes are suitable for sucking blood, but due to degradation, the male mosquito can only feed on nectar and plants juice [4-5].It usually takes $5 \mathrm{~min}$ for female mosquito to suck blood from the beginning to the end, while the entire sucking process is done with the unconsciousness of people. The specific function of mosquito mouth is determined by the special structure of its mouthparts.

The head of female culex was got and soften in alcohol, and then it was put in wintergreen oil to soak transparently about 10 min [6].

One to two drops of Arabic gum was put into the copper column. At the same time, the part of mosquito mouthpart which is connected to the head was adhered on the copper cylinder for the convenience of anatomy.

The mosquito mouthparts was incised and one hand holds the joint of the head and chest of the cicada, then use the anatomy needle to pick out the mouth needle from the lip groove of the lower lip back (be careful not to pick off) and separate it gently. The two thick ones outsides are the 
mouth needles of upper jaw, and the central thinner pair is mouth needle of two tightly embedded lower jaws (usually embedded as one).

Use the mixture liquid of anhydrous ethanol and ether (volume ratio 1:1) to degrease the mouth needle of the upper and lower jaw for $1 \mathrm{~h}$, and then use anhydrous ethanol dehydrate $1 \mathrm{~h}$ and dry naturally. These are used to make specimens for scanning of electron scanning microscope with gold sprayed.

Measure the characteristic of the non-smooth surface in mouthpart of upper and lower jaw (microns), and use the television camera stereoscopic microscope to observe the morphological structure of each part. Select the respective characteristics of the site sampling, and use SBC - 12 small ion sputtering apparatus spraying gold with gold thickness of about $20 \mathrm{~nm}$ [7], so that the electric discharge phenomena caused by uneven spray of gold or too thin layer can be avoided. Then use JMS - 5600LV scanning electron microscope scanning on anatomical mosquito mouthparts with the accelerating voltage $0 \sim 30 \mathrm{kV}$, resolution of $3.0 \mathrm{~nm}$, magnification of $30 \sim$ 350000 times and ZEISS Discovery V1 postures microscope to observe and video recorded [8].

\section{Observation of the Structure}

Mouthpart. The mouthpart of female mosquito is composed of six bundles of stitches wrapped inside by the outer sheath formed by the extension of the lower lip, and the outer sheath is the beak with tube shape but metameric. The front of the beak concaves into groove, and its top part presents the shape of lip. The length of beak is $3 \mathrm{~mm}$, the outside diameter is $60 \mu \mathrm{m}$ and the inside diameter is $25 \mu \mathrm{m}$. The appearance of the beak presents slender structure like grain and full of agnail, which looks like a mace (Fig. 2.1).
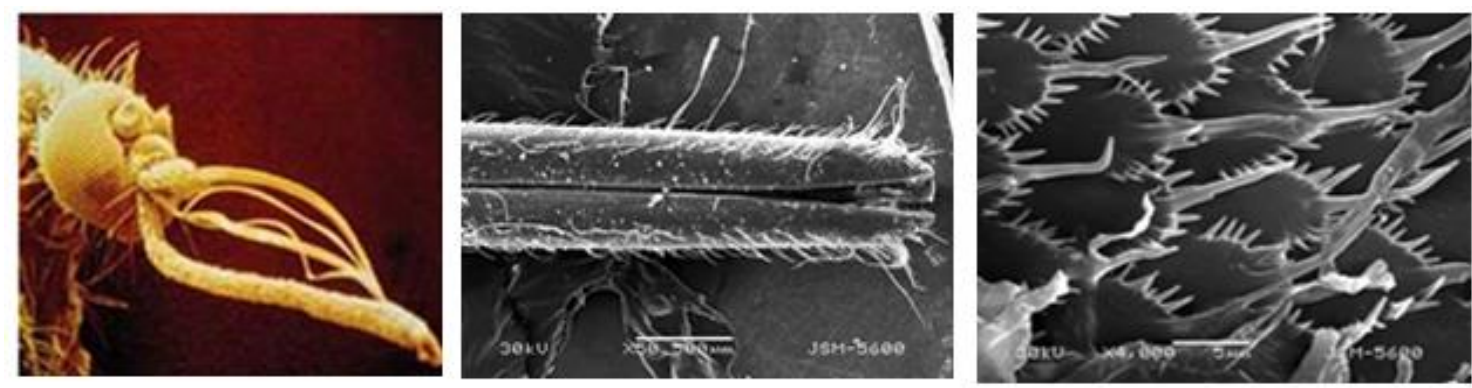

Figure 2.1. The appearance of mosquito mouthparts

There are six thin mouth needles (upper lip, a pair of upper and lower jaw, tongue) in the beak. Upper inside lip, tongue, upper jaw and lower jaw form the thorn organ, and the lower lip is only to protect these thorn organs. Located below the upper lip, tongue keeps the open bottom closed up together with the upper jaw to constitute a food tube. Tongue itself has a thin tube located in the central part of the tongue and link up with the salivary duct to inject saliva fluid. Below the tongue is the lower jaw.

Upper Jaw. The upper jaw is a pair of coarser mouth needle, which is a tool used to cut skin before sucking the blood. The front is thin, smooth, and the end is wide like a sword. There are 25 to 30 sawteeth at the end. The cross profile of the saw teeth is inequilaterally triangle. The sawteeth are regularly arranged on the upper jaw, and its span presents the trend of monotone increasing (figure 2.2).

There is a groove in the inner wall of the two upper jaws, and mouth needle is jogged together with groove ridge and can only slide up and down but can't be separated.

The sawtooth triangle in the top of mouth needle of upper jaw has the structure of barbs, and it is used to fix its position in the tissue in order to prevent its separation from the organization when the mouth needle reverse caused by the muscle contraction. 
Lower Jaw. Below the tongue is lower jaw. It is a pair thin mouth needle, and the front is thin and smooth, and the end is narrow with thin knife shape. There are about $13 \sim 15$ sawteeth in it. It plays a role in piercing the skin after the upper jaw cut the skin, and it is the main organ to do this.

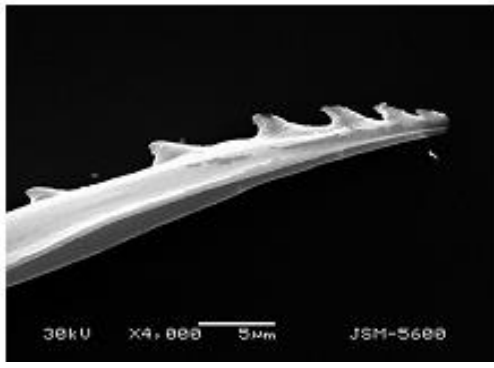

Figure 2.2 The images of electron microscope showing mosquito mouthparts palate

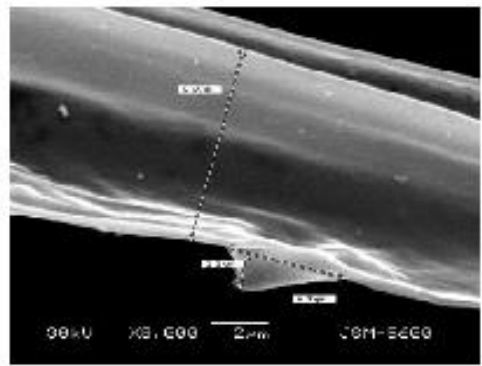

(a)

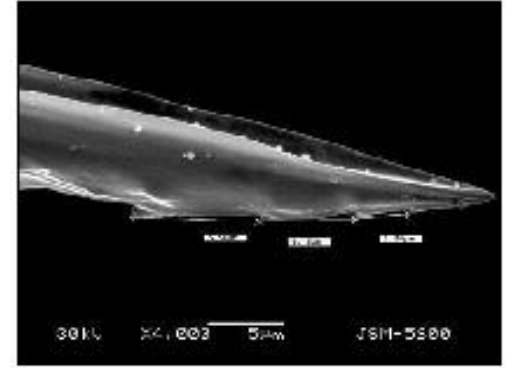

(b)

Figure 2.3 the mosquito jaw measurement figure [9]

The mouth teeth in the lower jaw of mosquitoes also present a typical non-smooth zigzag. The cross section of sawteeth presents a non-equilateral triangle inverse structure, and the structure plays a role in fixing when the mosquito repeatedly saw skin (as shown in Fig. 2.3 a).

The arrangement of the non-smooth saw structure in the lower jaw is also regular. The distance between sawteeth varies with the trend of monotone increasing (as shown in Fig. 2.3 b) [9].

The structure parameters of the mouth needle of the lower jaw: diameter, which is from sawtooth at the bottom of the triangle to the edge of jaws groove, is 6.69 microns; the lower side of the triangle sawteeth is $4.05 \mu \mathrm{m}$; the height is $1.17 \mu \mathrm{m}$; The ratio of mouth needle diameter, sawteeth bottom edge and sawteeth height is 27:16:4.

On the same lower jaw needle, the distance between the three consecutive point is: $3.56 \mu \mathrm{m}$, $6.45 \mu \mathrm{m}, 8.56 \mu \mathrm{m}$, respectively, and the ratio is 7:13:17 [9].

In each jaw inside, there are two longitudinal grooves. Two lower jaws become two pipelines. The front one which is slightly rough is food canal, and the rear one which is slightly thin is salivary canal, through which saliva flow into the organization. When the two mouth needles of upper jaw pierce the same depth, the chimerical together with one pair of lower jaws needles pierces immediately $[10,11]$, formed an structure that can be operated by as one integration.

Two longitudinal grooves in the jaw line joint each other and form tubular thin-walled structure.

\section{Discussion and Analysis}

This study found that the mouth needle of mosquitoes presents typical flexible non-smooth morphology: the front of jaws is thin and smooth and the end is knife shaped; the end of the surface is regularly arranged with the sawteeth structure of non-equilateral triangle shape, and jaws inside are grooves, a pair of the upper and lower jaw joint mutually, which constitutes a thin-walled tubular structure. Some sucking mouthparts of such as the mosquito have the characteristics of reducing resistance. The key lies in the "O" shaped tubular structure of the thin-wall hollow of its sucking mouthparts, and the outer surface of the thin-walled tubular structure has typical non-smooth form. It is the non-smooth shape joint of the special structure and its wall that realized the function of decreasing resistance and the painless of sucking.

The mosquito's mouthpart consists of six mouth needles and the effect of cutting skin only depends on the jaw. When mosquito is sucking blood, the sharp front of mouthparts pushes in like oil drill. Therefore, only a small wound appeared, and the stimulation to the nerve is not big and caused pain is very small. The sawteeth structure on the surface of the mouth needle of the jaws has the effect of sawing skin and hosting organization. It is this non-smooth morphology in the needle that makes the contact area with the skin is less when it is piercing in, and the nerve stimulation is weak that reduce the feeling of pain. When it reduces to the pain threshold, people will not feel pain. 
The agnail plays a role in fixing the position to make sure that mouth needles will not exit from the host organization. That is why mosquitoes can suck the blood of human and animals without their pervasive.

In the process of sucking, the non-smooth structure of jaws reduces the contact area between the mouth and the host, and hole exists in its contact surface. It is easy to form no muscle area on the surface of needle and skin. There are air membrane between surface of needle mouth and muscle, which reduces the adhesion between muscle tissue and mouthpart wall as well as reduces the friction factor, thus reducing the friction.

In the pierce process of mouthparts, the non-smooth surfaces makes host organization contact interface that relatively moves with mouthparts surface have micro-vibration in the vertical direction of the forward. The host organization is constantly affected by the interaction of the pros and cons force in the vertical interface. Micro vibration accelerate the escape of tissue fluid, and it is added to the hole providing a weak shear layer, which has played an important role in increasing lubrication and reducing adhesion , and forming the model which has the same resistance reduction mechanism of rolling bearing, turning dry friction into lubrication friction. Friction factor decreases causing the reduction of friction and reduces the stimulation to nerve, which can reduce the pain.

When the mosquito is sucking, host tissue is stimulated to tension, which is composed of two parts. The first one is due to the viscosity of the skin tissue, which is caused by tension created when the surface of mouthpart contacts with the skin, i.e. passive tension. The second one is that muscle contraction causes active tension which is reflex due to the stimulation of the skin. The weaker needle stimulation to the skin, the smaller contraction tension. While the reduction of shrinkage can cause the reduction of local tissue density, and its viscosity and the contact area with the needle also decreases relatively, thus reducing friction factor and the friction. Consequently, the resistance drops greatly.

\section{Simulation Bionic Non-Smooth Morphology Of Mosquito Mouthpart}

Based on the non-smooth morphology of the mouth needle of mosquito mouthpart jaws, the non-smooth surface was modeled. The imitation of the non-smooth morphology of mosquito was processed into the syringe apparatus which is $25 \mathrm{~mm}$ away from the front, making it a zigzag and corrugated type. The bionic non-smooth model is shown as figure 4.1. And experimental analysis of injection resistance reduction rate was conducted.

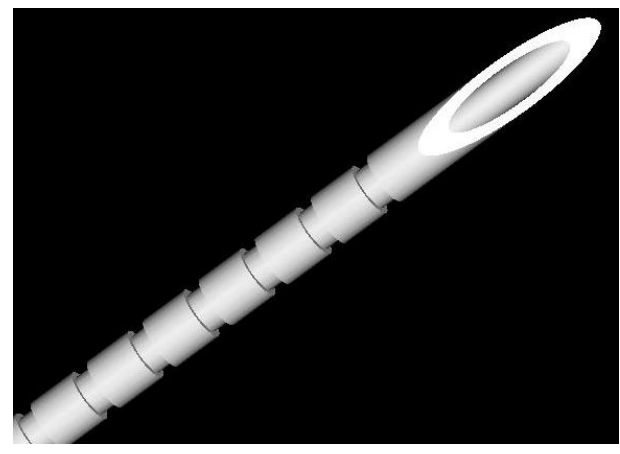

(a) sawteeth non-smooth model

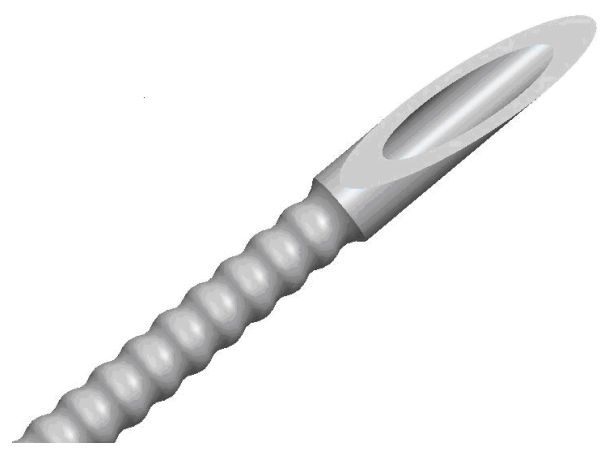

(b) corrugated non-smooth model

Figure 4.1. the bionic painless injectors with non-smooth model

When needle is piercing organization, friction is produced between needle and skin. Through the determination of frictional resistance, the value of the drag reduction can be quantitatively analyzed. Consequently, the non-smooth morphology with the best effect of reducing resistance was found out. The drag reduction rate is used to study the drag reduction characteristics, so as to determine the effect of resistance variation when inserting needle with non-smooth surface of bionic needles into the needle resistance change, as shown in Fig. 4.2. 
By comparing the reduction rate, it is further explained that all of the bionic pieces tested have basically achieved drag reduction effect on the entire depth of the needle, except for the third needle which has a phenomenon of increasing resistance in certain region. The fourth needle has the best effect of resistance reduction, with maximum drag reduction rate of $50.49 \%$ and average drag reduction rate of $45.17 \%$.

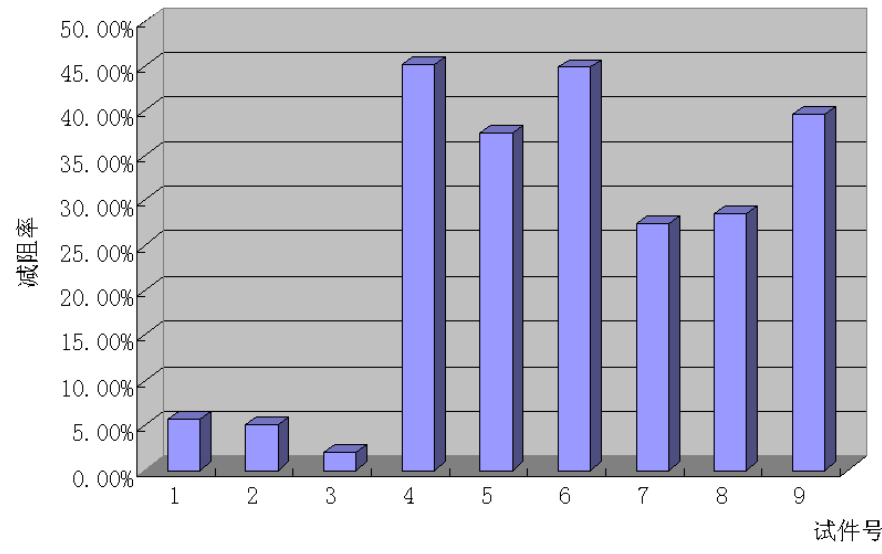

Figure 4.2. comparison chart of drag reduction rate
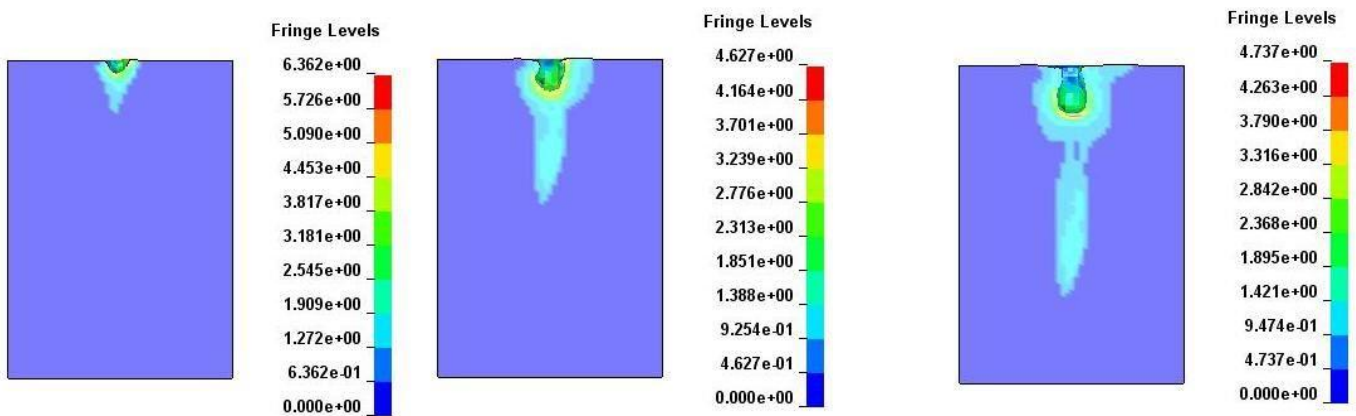

(a) smooth shape needles stress nephogram
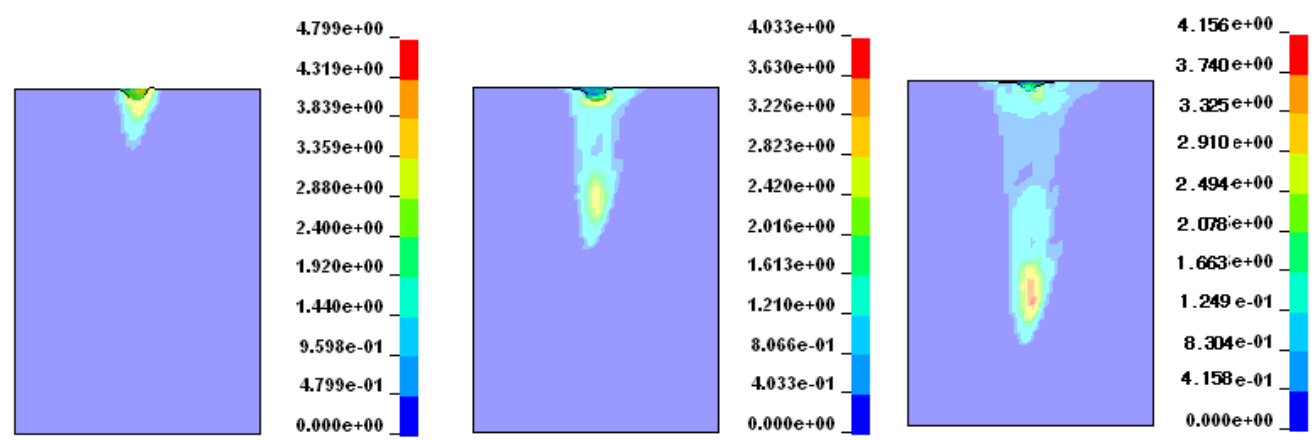

(b) the sawtooth needles stress nephogram
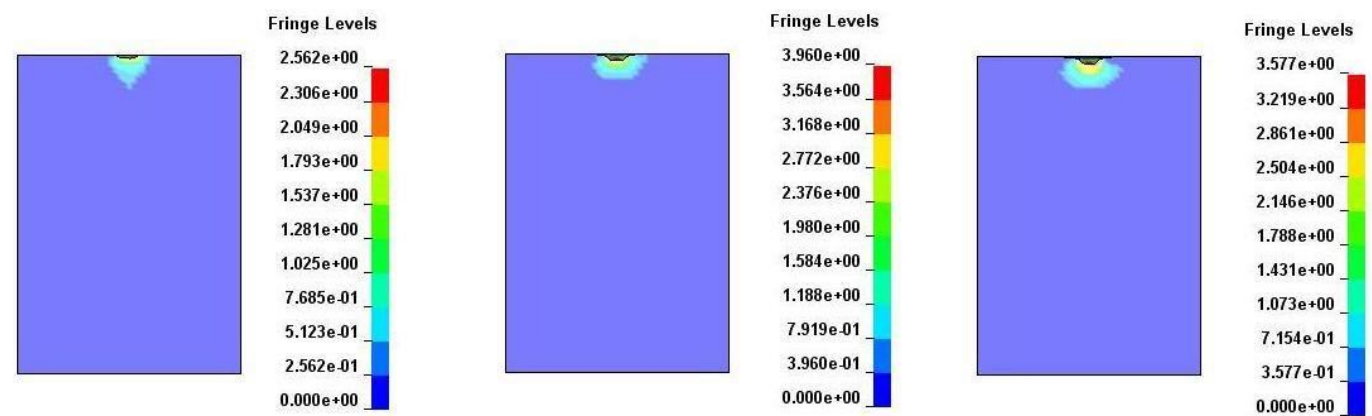

(c) The stress nephogram of corrugated-shaped needle

Figure 4.3. The stress nephogram of symmetry plane of smooth needle and bionic needle in the process of injection at three different times 
Fig. 4.3 is the stress cloud which present the symmetric plane of the soft tissue at three different times during the injections with the smooth needle, the bionic tooth shaped needle and the bionic ripple needle, respectively.

The smaller the stress, the smaller the simulation to the nervous system within the soft tissue, and also the better pain relief effect. The smaller the soft tissue by the needle penetration in the process of injection velocity, the smaller the stimulation nerve suffered and slighter the pain feeling. In the process of needle penetration, resistance causes the change of internal energy. The smaller internal energy, the smaller resistance. At the same time, the smaller the stimulus of the nervous system and less pain. It can be seen from the diagram of corrugated shape that the effect of pain relief using bionic needle is better than that using sawteeth, which is accord with the experimental results.

Experimental study shows that the syringe needle with non-smooth surface imitating the mouthpart of mosquito can reduce resistance and have the effect of painless injection, compared with that of the needle with smooth surface.

\section{Acknowledgements}

Key project of Jilin province science and technology development, number: 20120448

\section{References}

[1] L.L. Chen,J.C. Wang Syringe needle surface bionic nonsmooth form drag reduction tests in April 2006(In Chinese)

[2] X.Q. Kong, C.W. Wu .Measurement and prediction of in sertion force the mosquito fascicle penetrating into humanskin. J Bionic Eng, (2009), 6(2):P:143(In Chinese)

[3] X.Q. Kong, C.W. Wu. Numerical simulation of the mosquito's mouth needle human skin process. Journal of mechanics and practice, (2010), 32 (2): p:90-94(In Chinese)

[4] Aoyagi S, Izumi H. Development of micro lancet needle made of biodegradable polymer for medical treatment. Institute of electrical engineers of Japan, (2007), 127(2): p: 342.

[5] X.J. Liu, D.M. Su, Q.S. Yang. Insect morphological taxonomy. Shanghai: Fudan university press, 1985. (In Chinese)

[6] X.L. wu, X.B. huo, X.J. Practical medical entomology experiment technology of shandong science and technology publishing house, 1999 In the first edition.(In Chinese)

[7] G.Y. ling, Z.W. guo. Insect SEM sample Making Problems Electron Microscopy Society, 2003, 22 (6): P: 668. (In Chinese)

[8] Yangshen branches, Qinger ji. Insect scanning electron microscope sample drying method .Fujian Agricultural University, 2001,2 (30): 262-265.

[9] Beijing Agricultural University entomology editor of The General Theory (the book) Agricultural Publishing (1981).4,p: 43(In Chinese)

[10]Richards, RG Davies Amendment Jie liu xin, Deming su, Qingshuang yang. Islamic female translated entomology tutorial. Higher Education Press (1987), 12, p:24

[11]S.T. Gu. Based on Greenfield Valley sucking insects biomimetic syringe (MS. Jilin University, China 2008.) (In Chinese) 